Zusammenhangs zwischen der Nachhaltigkeitsberichterstattung und der Nachhaltigkeitsperformance erklären können.

Aufgrund der zunehmenden Bedeutung qualifizierter Mitarbeitender besteht für Unternehmen die Notwendigkeit, auf dem Arbeitsmarkt als attraktiver Arbeitgeber wahrgenommen zu werden. Die Relevanz der Arbeitgeberattraktivität wird in der wissenschaftlichen Literatur bereits stark thematisiert; die Identifikation von Determinanten der Attraktivität eines Arbeitgebers aus Sicht der aktuellen Mitarbeitenden im Unternehmen wurde bisher aber weitgehend vernachlässigt. Der Beitrag von Manfred Brubn, Verena Batt und Benjamin Flückiger widmet sich dieser Forschungslücke. Die Autoren stellen ein Untersuchungsmodell zum Aufbau von Arbeitgeberattraktivität vor; anschliessend wird die Frage, ob die identifizierten Grössen bei aktuellen Mitarbeitenden tatsächlich zur Entstehung der Arbeitgeberattraktivität beitragen, auf Basis einer empirischen Untersuchung thematisiert. Die Ergebnisse deuten darauf hin, dass Arbeitgeberattraktivität bei Mitarbeitenden vor allem durch weiche Faktoren, wie z.B. den Führungsstil und die Arbeitsplatzqualität, sowie durch Imagefaktoren beeinflusst wird. Harte Faktoren, wie z.B. die Entlohnung oder Entwicklungsmöglichkeiten, sind gemäss der empirischen Studie nicht relevant, wenn es um die Beurteilung der Arbeitgeberattraktivität geht.

Was den Herausgeberkreis der Zeitschrift «Die Unternehmung» anbelangt, möchte ich Sie auf zwei Änderungen hinweisen: Georg von Krogh (ETH Zürich) ist per Anfang des Jahres von seinem Amt als Mitherausgeber zurückgetreten. Im Namen aller Herausgeber danke ich ihm für sein wertvolles Engagement zugunsten der Zeitschrift. Begrüssen möchte ich gleichzeitig Markus Gmür (Universität Fribourg), der die Nachfolge von Georg von Krogh antreten wird. Alle Herausgeber freuen sich auf die Zusammenarbeit mit Markus Gmür und wünschen ihm einen guten Start in diesem Kreis.

Zudem freut es mich, Ihnen in diesem Heft die Übergabe des «Best Paper Award 2012» an Frank Piller, Alexander Vossen und Christoph Ihl bekannt zu machen. In der Laudatio von Manfred Bruhn werden die Gründe für die Wahl dieses Aufsatzes dargelegt. Der «Best Reviewer Award 2012» für ein besonders hochwertiges Gutachten geht an Dennis Schoeneborn. Im Namen der Herausgeber gratuliere ich den Preisträgern herzlich. Allen Gutachterinnen und Gutachtern der vergangenen Jahre, die zur Sicherstellung der wissenschaftlichen Qualität der Zeitschrift beigetragen haben, gilt ein grosses Dankeschön.

Ich wünsche Ihnen eine anregende Lektüre

Ihr Dieter Pfaff

\title{
Best Paper Award
}

Der Beitrag «From Social Media to Social Product Development: The Impact of Social Media on Co-Creation of Innovation» von Frank Piller, Alexander Vossen und Christoph $I h l$ wird aus folgenden drei Gründen mit dem «Best Paper Award 2012» von «Die Unter- 
nehmung» ausgezeichnet: Erstens behandelt der Beitrag mit der Untersuchung des Einflusses von Social Media auf Customer Co-Creation ein für die Wissenschaft und die Unternehmenspraxis derzeit äusserst relevantes Thema. Zweitens ergänzt er durch die Verbindung dieser beiden zentralen Entwicklungen der letzten Jahre die bisherige Forschung um innovative Inhalte und schlägt drittens eine Brücke zwischen Theorie und Praxis.

Zunächst ist die Relevanz des Themas hervorzuheben. Eine zunehmende Anzahl an Unternehmen versucht, die Konsumenten in den Prozess der Ideengenerierung und der Produktgestaltung mit einzubinden, um eine so genannte «Customer Co-Creation» ihrer Produkte und Services zu erreichen. Als Beispiele aus der Unternehmenspraxis sind hierbei die Plattformen der Firmen Starbucks «My Starbucks Idea», Tchibo «Tchibo Ideas» oder Google «Google Demo Slam» anzuführen. Die Etablierung von Social Media-Plattformen hat Unternehmen im Kontext der Co-Creation eine Vielzahl an Möglichkeiten eröffnet, eine grosse Anzahl an Nutzern in den Prozess der Customer Co-Creation zu integrieren. Bislang fehlt es jedoch weitgehend an einer Systematisierung der verschiedenen Formen von Customer Co-Creation sowie einer detaillierten Analyse des Einflusses von Social Media auf die jeweiligen Formen. Der Beitrag von Frank Piller, Alexander Vossen und Christoph I $h l$ entwickelt eine solche Systematisierung der verschiedenen Formen der Kundenintegration in den Innovationsprozess und zeigt zudem für die einzelnen Formen jene Möglichkeiten auf, die mittels Social Media geboten werden; aber diskutiert auch die Gefahren, die hierbei entstehen können. Somit liefert der Beitrag wesentliche Erkenntnisse bezüglich des Einflusses von Social Media auf Customer Co-Creation und ermöglicht die Ableitung erfolgsrelevanter Handlungsimplikationen für die Praxis.

Weiterhin zeichnet sich der Beitrag in mehrfacher Hinsicht durch seine Innovativität aus. Zunächst wird - aufbauend auf der bestehenden Literatur - eine relevante Typologie der Kundenintegration in den Innovationsprozess abgeleitet. Hierbei werden anhand der Art der Information als auch der Art der Austauschbeziehung vier verschiedene Typen von Customer Co-Creation identifiziert und der Einfluss von Social Media diskutiert. Die Autoren zeigen, dass der Einsatz von Social Media im Rahmen der Customer Co-Creation zu einer Verschiebung der Austauschbeziehungen führen kann - von sozialen zu ökonomischen Austauschbeziehungen und vice versa. Die systematische Verbindung von Customer Co-Creation und Social Media stellt eine wichtige Ergänzung der bisherigen Forschung dar. Weiterhin wird durch die Ableitung einer Vielzahl weiterführender Fragestellungen eine gelungene Grundlage für zukünftige Forschungsarbeiten in diesem Bereich geschaffen.

Abschliessend verbindet der Beitrag Theorie und Praxis, indem eine Klassifizierung der verschiedenen in der Praxis existierenden Formen von Customer Co-Creation ermöglicht wird und konkrete Hinweise zum Einsatz von Social Media im Rahmen von Customer Co-Creation gegeben werden. In diesem Kontext ist vor allem die gelungene Darstellung mittels geeigneter Praxisbeispiele zu betonen. Die Autoren leiten konkrete Handlungsimplikationen für Unternehmen ab und zeigen die Möglichkeiten als auch die Risiken für ein Social Media-Engagement im Kontext der Customer Co-Creation auf. Somit bietet dieser sehr gelungene Beitrag sowohl für die weiterführende Forschung als auch die Unternehmenspraxis zentrale sowie gut strukturierte Erkenntnisse.

Prof. Dr. Manfred Brubn, Universität Basel 
Die Herausgeber der Zeitschrift «Die Unternehmung» verleihen

\author{
Prof. Dr. Frank Piller \\ Alexander Vossen \\ Dr. Christoph Ihl
}

für ihren im Heft 1/2012 publizierten Beitrag

«From Social Media to Social Product Development:

The Impact of Social Media on Co-Creation of Innovation»

den

\title{
"Best Paper Award 2012"
}

Der Award wird verliehen für Publikationen mit einer besonders hohen wissenschaftlichen Güte und Tragweite. Die Preisträger haben ein wichtiges Thema von allgemeiner Relevanz behandelt, Innovatives präsentiert und eine Brücke zwischen Forschung und Praxis geschlagen. Somit haben sie einen bedeutenden Beitrag zur Förderung der wissenschaftlichen Qualität und des Renommees der Zeitschrift «Die Unternehmung» geleistet. Dafür sind die Herausgeber den Preisträgern sehr dankbar.

Zürich, im März 2013

Prof. Dr. Dieter Pfaff Geschäftsführender Herausgeber 


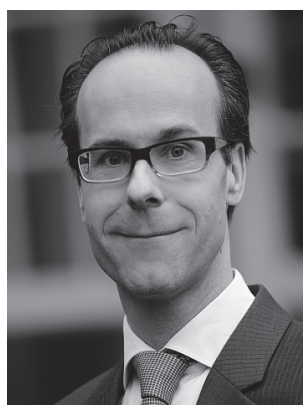

Frank Piller, Prof. Dr., forscht seit vielen Jahren über die Gestaltung kundenzentrierter Innovations- und Wertschöpfungsprozesse und die Nutzung externen Wissens für den Innovationsprozess. Er ist seit 2007 Lehrstuhlinhaber für Technologie- und Innovationsmanagement an der RWTH Aachen. Hier verantwortet er auch als Studienleiter den gemeinsamen Executive MBA von RWTH und Fraunhofer Gesellschaft. Er ist weiterhin Co-Direktor der Smart Customization Group am MIT Media Lab des Massachusetts Institute of Technology, wo er von Ende 2004 bis Anfang 2007 an der MIT Sloan School of Management arbeitete. Frank Piller promovierte zum Thema "Mass Customization“ an der Universität Würzburg und habilitierte 2004 zum Thema „Kundenintegration im Innovationsprozess“ an der TU München am Lehrstuhl IOM von Prof. Ralf Reichwald. Aktuelle Forschungsgebiete seines Lehrstuhls an der RWTH sind die organisationale Erfolgsfaktoren für Open Innovation, Designfaktoren (insb. Anreizstrukturen) von Co-Creation, Technologietransfer und die Formation informaler Handlungsräume für Innovation. Prof. Piller ist Autor vieler Bücher, Studien und Artikel. Neben guter wissenschaftlicher Forschung sind ihm auch eine engagierte Lehre sowie der Erkenntnistransfer in die Praxis besondere Anliegen. Als wissenschaftlicher Partner der Managementberatung Competivation, Beirat von Unternehmen wie Hyve, Dialego oder Corpus.e und durch Engagements im Bereich Executive Education bringt er deshalb seine Forschung in Grossunternehmen wie in innovative KMU.

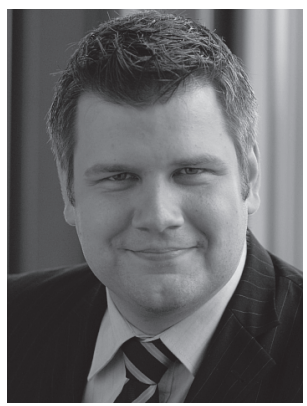

Alexander Vossen ist seit Januar 2009 Wissenschaftlicher Mitarbeiter und Doktorand am Lehrstuhl für Technologie und Innovationsmanagement. Seine Forschungsinteressen liegen im Bereich des kreativen Konsumentenverhaltens, insbesondere im Bereich Anreize und deren Auswirkungen auf das tatsächliche Verhalten von Konsumenten. Vor dem Hintergrund dieser Fragestellungen untersucht er in seiner Dissertation von Unternehmen veranstaltete Ideenwettbewerbe mit Konsumenten.

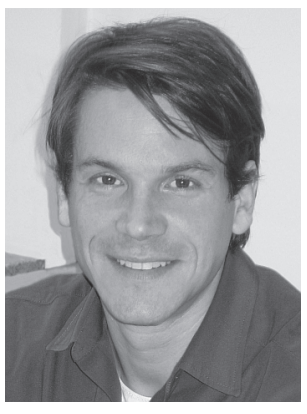

Christoph Ihl, Dr., ist seit Juli 2009 Habilitand am Lehrstuhl für Technologie- und Innovationsmanagement der RWTH Aachen. Zuvor hat er an der Technischen Universität München zum Thema Mass Customization und Konsumentenverhalten promoviert. Er hat ein Studium des Wirtschaftsingenieurwesens in der Fachrichtung Maschinenbau an der Technischen Universität Berlin sowie ein MBA-Programm an der University of British Columbia in Vancouver absolviert. Die Forschungsinteressen von Christoph Ihl liegen der Untersuchung von offenen und verteilten Innovationprozessen. Hierbei setzt er die folgenden Schwerpunkte: (1) Geeignete formelle und informelle Organisationstrukturen in Unternehmen; (2) Einbin- 
dung von Kunden- und Nutzer-Communities in die Produktentwicklung; (3) Technologietransfer zwischen Wissenschaftlern und Unternehmen. In diesen Themenbereichen arbeitet er auch immer wieder mit Unternehmen in wissenschaftlich fundierten Beratungsprojekten zusammen.

\section{Reviewer Award}

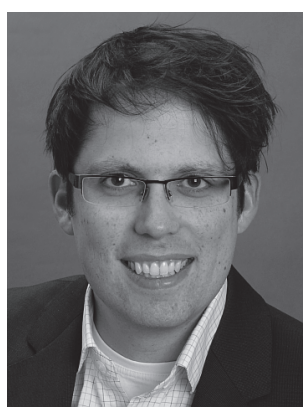

Dennis Schoeneborn, Dr., ist seit Juli 2008 Oberassistent am Institut für Betriebswirtschaftslehre der Universität Zürich. Zuvor studierte er Medienmanagement in Hannover und London (LSE). Anschliessend absolvierte er das Doktorat in Betriebswirtschaftlehre an der Bauhaus-Universität Weimar. Darüber hinaus sammelte er Praxiserfahrung als Managementberater für The Boston Consulting Group (BCG). Seine Forschungsschwerpunkte sind Organization Studies, Organizational Communication, Computer-Mediated Communication und Corporate Social Responsibility (CSR). Seine Arbeiten wurden in internationalen Fachzeitschriften der Organisations- und Managementforschung veröffentlicht (u.a. Journal of Management Inquiry, Management Communication Quarterly und Organization Studies). Im Jahr 2013 ist er gemeinsam mit François Cooren und Tim Kuhn Ausrichter des thematischen Tracks zu Organizational Communication auf dem European Group of Organizational Studies (EGOS) Colloquium in Montréal, Kanada. 